Classification

Physics Abstracts

74.70B

\title{
Influence des traitements de fluoration sur les propriétés de transport des céramiques $\mathrm{YBa}_{2} \mathrm{Cu}_{3} \mathrm{O}_{7-\delta}$
}

\author{
C. Magro, J. M. Heintz, A. Tressaud, J. P. Bonnet et J. Etourneau \\ Laboratoire de Chimie du Solide du CNRS, Université de Bordeaux 1, 351, cours de la \\ Libération, 33405 Talence Cedex, France
}

(Reçu le 25 mars 1991, accepté le 17 juillet 1991)

\begin{abstract}
Résumé. - La dégradation des céramiques $\mathrm{YBa}_{2} \mathrm{Cu}_{3} \mathrm{O}_{7-\delta}$ élaborées par voie solide est essentiellement due à des mécanismes de carbonatation. L'existence d'une couche de carbonates résiduels à l'interface solide/gaz constitue l'origine de la diminution sensible de la densité de courant critique $J_{\mathrm{c}}$, dans le cas de matériaux exposés plusieurs mois à l'air. Des traitements de fluoration réalisés sur nos céramiques immédiatement après le cycle de frittage ont montré une amélioration de $J_{\mathrm{c}}$ ainsi qu'un ralentissement du vieillissement de ces matériaux.

Abstract. - The degradation of $\mathrm{YBa}_{2} \mathrm{Cu}_{3} \mathrm{O}_{7-\delta}$ ceramics obtained by solid state sintering is known to be due to carbonation processes. The presence of a carbonate layer at the solid/gas interface contributes to decrease the critical current density after several months ageing in air conditions. Fluorination treatments carried out on $\mathrm{YBa}_{2} \mathrm{Cu}_{3} \mathrm{O}_{7-\delta}$ ceramics immediately after the sintering process increase noticeably $J_{c}$ by a curing effect of the surface. On the other hand a real passivation against ageing process is observed.
\end{abstract}

\section{Conditions de préparation et de fluoration.}

Les céramiques $\mathrm{YBa}_{2} \mathrm{Cu}_{3} \mathrm{O}_{7-\delta}$ ont été élaborées à partir d'un lot de poudre de provenance Rhône-Poulenc SU 57. Un procédé classique de frittage a été mis en œuvre : après deux cycles de traitement sous oxygène à $920^{\circ} \mathrm{C}$ pendant $2 \mathrm{~h}$, les céramiques étaient réoxydées à $400{ }^{\circ} \mathrm{C}$ pendant $24 \mathrm{~h}$, température de réoxydation considérée comme optimale d'après les données thermodynamiques [1].

Le traitement de fluoration a été réalisé selon une méthode précédemment mise au point au laboratoire [2]. Les expériences étaient réalisées sous fluor pur à des températures comprises entre $20^{\circ} \mathrm{C}$ et $100^{\circ} \mathrm{C}$ pendant des cycles de $20 \mathrm{~h}$; il est à noter que le traitement sous fluor est effectué à basse température afin de limiter la prise de masse relative à la formation d'une couche amorphe (oxy)fluorée à moins de $1 \%$.

Les valeurs de $J_{c}$ des céramiques ainsi élaborées ont été déduites de mesures de susceptibilité alternative entre la température critique et $80 \mathrm{~K}$ (modèle de Bean [3]).

\section{Amélioration de $J_{c}$ par un traitement de fluoration.}

La figure 1 montre l'évolution de $J_{\mathrm{c}}$ avec la température dans le cas des céramiques ayant subi 


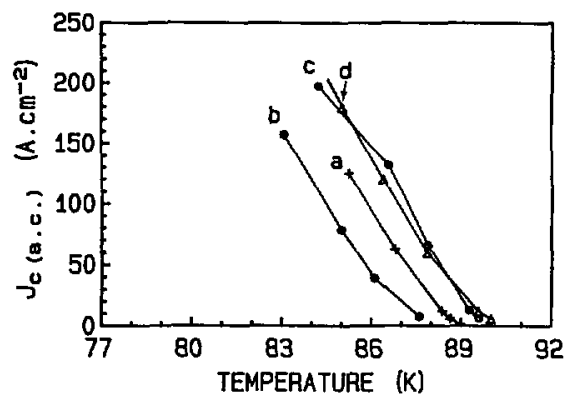

Fig. 1. - Evolution thermique de $J_{c}$ pour une céramique ayant subi les traitements successifs : a) 2 frittages $920{ }^{\circ} \mathrm{C}$; b) a $+\mathrm{O}_{2} 400{ }^{\circ} \mathrm{C}$; c) $\mathrm{b}+\mathrm{F}_{2} 100{ }^{\circ} \mathrm{C}$; d) $\mathrm{b}+\mathrm{F}_{2} 20^{\circ} \mathrm{C}$.

deux traitements thermiques. Le traitement de fluoration a été effectué quelques jours seulement après la réoxydation à $400{ }^{\circ} \mathrm{C}$.

La dégradation du courant critique observée après un traitement de réoxydation à $400{ }^{\circ} \mathrm{C}$ (courbe b) de céramiques frittées à $920^{\circ} \mathrm{C}$ (courbe a) résulte vraisemblablement de la présence de la couche amorphe de carbonates résiduels à l'interface solide/gaz [4]. La cinétique de diffusion de l'oxygène à travers celle-ci devient alors plus faible que celle de la diffusion de l'oxygène à l'intérieur du grain ; il est probable alors qu'une homogénéisation de la teneur en oxygène intragranulaire se produise, si bien que $J_{\mathrm{c}}$ diminue [5].

Un traitement de fluoration de ces céramiques réoxydées provoque une notable augmentation de $J_{\mathrm{c}}$. La comparaison des courbes $\mathrm{c}$ et $\mathrm{d}$ montre que le résultat est le même quelle que soit la température de fluoration dans le domaine $20 \leqslant T_{\mathrm{F}_{2}} \leqslant 100^{\circ} \mathrm{C}$.

L'amélioration des propriétés supraconductrices des céramiques $\mathrm{YBa}_{2} \mathrm{Cu}_{3} \mathrm{O}_{7-\delta}$ par un procédé de fluoration peut être associée aux deux phénomènes suivants:

- nettoyage de la surface du matériau par décomposition des carbonates et des impuretés semi-conductrices à base d'ions $\mathrm{Cu}^{2}+$ localisées à la surface et aux joints de grains comme en témoigne la disparition, après traitement sous fluor, des signaux RPE dus aux ions $\mathrm{Cu}^{2}+$ localisés [6] ;

- la formation d'une couche amorphe (oxy)fluorée totalement stable dans les conditions ambiantes et dont l'épaisseur a été évaluée par AES comme étant de l'ordre de quelques milliers d'angströms.

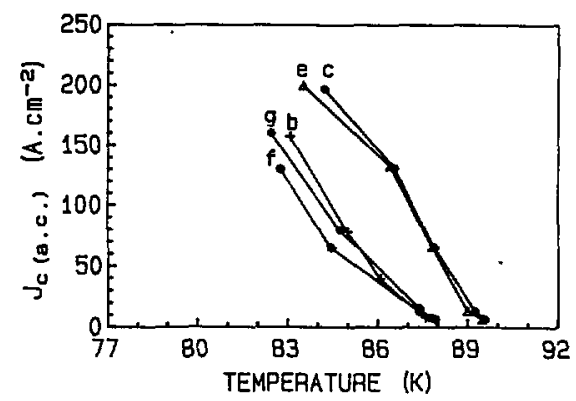

Fig. 2. - Influence de la fluoration sur la variation de $J_{\mathrm{c}}$ avec le temps : b) céramique frittée $920^{\circ} \mathrm{C}$ et réoxydée à $400^{\circ} \mathrm{C}$ (id. Fig. 1); c) b $+\mathrm{F}_{2} 100^{\circ} \mathrm{C}$ (id. Fig. 1); e) $\mathrm{c}$ après un vieillissement de 3 mois à l'air ; $\mathrm{f}) \mathrm{b}$ après un vieillissement de 3 mois à l'air ; g) $f+\mathrm{F}_{2} 100^{\circ} \mathrm{C}$. 


\section{Influence des traitements de fluoration sur le vieillissement.}

La figure 2 montre l'évolution de $J_{\mathrm{c}}$ avant et après traitement de fluoration.

Lorsqu'une céramique réoxydée à $400{ }^{\circ} \mathrm{C}$ (courbe b) a été exposée pendant 3 mois au contact de l'atmosphère (courbe $f$ ), une diminution considérable de $J_{c}$ est observée. De nombreux travaux ont montré que la dégradation du matériau se produit à partir de la couche de pollution de surface contenant les espèces carbonatées. Un traitement de ce même échantillon sous fluor pur à $100^{\circ} \mathrm{C}$ (courbe $\mathrm{g}$ ) améliore le courant critique sans toutefois permettre de retrouver les valeurs initiales de $J_{c}$. La barrière de diffusion formée par la couche de carbonates limite donc l'effet nettoyant de la fluoration à la surface des grains.

En revanche, un matériau réoxydé à $400^{\circ} \mathrm{C}$ puis fluoré à $100^{\circ} \mathrm{C}$ (courbe c) ne présente pas de dégradation notable de $J_{c}$ après 3 mois au contact de l'atmosphère (courbe e). Il semble donc que la couche amorphe (oxy)fluorée de surface ait ralenti considérablement la détérioration de la céramique au cours du temps en protégeant le matériau de tout échange avec l'atmosphère.

\section{Conclusions.}

Un traitement de fluoration à basse température $\left(20 \leqslant T_{\mathrm{F}_{2}} \leqslant 100^{\circ} \mathrm{C}\right)$ effectué sur des céramiques supraconductrices $\mathrm{YBa}_{2} \mathrm{Cu}_{3} \mathrm{O}_{7-\delta}$ frittées par voie solide puis réoxydées à $400{ }^{\circ} \mathrm{C}$ conduit à la formation d'une couche de passivation amorphe (oxy)fluorée. Celle-ci élimine la couche amorphe de carbonates résiduels (à l'origine de la dégradation de $J_{c}$ ) et ralentit considérablement le processus de vieillissement du matériau en le protégeant de tout échange avec les agents extérieurs tels que $\mathrm{H}_{2} \mathrm{O}$ et $\mathrm{CO}_{2}$. Des études sont actuellement en cours en vue d'augmenter la teneur en oxygène des céramiques fluorées.

\section{Remerciement.}

Ce projet a été réalisé dans le cadre du programme CNRS «GS Matériaux Supraconducteurs » avec l'aide de Rhône-Poulenc Recherches et Alsthom-Alcatel Recherches.

\section{Bibliographie}

[1] Gallagher P. K., Lindemer T. B. et al., J. Am. Ceram. Soc. 72 (1989) 1775.

[2] Tressaud A., Chevalier B., Lepine B., Amine K., Lozano L., Marquestaut E., Etourneau J., Eur. J. Solid State Inorg. Chem. 27 (1990) 309.

[3] BeAn C., Rev. Mod. Phys. 36 (1964) 1.

[4] Heintz J. M., Magro C., Fröhlich K., Dordor P., Bonnet J. P., J. Less Common Metals 164, 165 (1990) 1377-1384.

[5] Alford N. Mc N., Clegg W. J., Harmer M. A., Birchali J. D., Kendall, K., Jones D. H., Nature 332 (1988) 3.

[6] Dance J. M., Tressaud A., Chevalier B., Darriet J., Etourneau J., Solid State Ionics 32-33 (1989) 1188. 